\title{
Determinación de partículas suspendidas totales durante cinco cruceros de investigación en la plataforma continental del Golfo de México
}

\author{
R. Sosa-Echeverría ${ }^{1}$, H. Bravo-Alvarez ${ }^{1}$, P. Sánchez-Alvarez ${ }^{1}$, R. Soto-Ayala ${ }^{2}$, \\ A. Alarcón-Jiménez ${ }^{1}$ y J.D.W-Kahl ${ }^{3}$ \\ ${ }^{1}$ Sección de Contaminación Ambiental, Centro de Ciencias de la Atmósfera, UNAM, \\ ${ }^{2}$ División de Ciencias Básicas, Facultad de Ingeniería, UNAM y \\ ${ }^{3}$ Department of Mathematical Sciences, University of Wisconsin-Milwauke \\ E-mails: hbravo@servidor.unam.mx,rodsosa@servidor.unam.mx
}

(Recibido: abril de 2005; aceptado: diciembre de 2006)

\begin{abstract}
Resumen
La Universidad Nacional Autónoma de México (UNAM), ha realizado estudios sobre la calidad del aire en cinco cruceros de investigación en el Golfo de México. El primero de ellos conducido en el verano de 1986, con la participación conjunta de la National Oceanic and Atmospheric Administration (NOAA) y la Sección de Contaminación Ambiental (SCA) del Centro de Ciencias de la Atmósfera (UNAM) y la Secretaría de Marina de México, el cual se enfocó a mediciones de la calidad del aire. Los cuatro cruceros realizados posteriormente: de septiembre-octubre del 2002, de noviembre-diciembre del mismo año, de septiembre-octubre de 2003 y de mayo-junio del 2004, respectivamente, se enfocaron, además de la calidad del aire, a investigaciones oceanográficas.

La caracterización de las partículas atmosféricas se llevó a cabo muestreando las partículas suspendidas totales (PST) mediante métodos que cumplen con las Normas Oficiales Mexicanas, así como con los Métodos de Referencia de la Agencia de Protección Ambiental de los Estados Unidos (USEPA) para partículas suspendidas totales (Método de Altos Volúmenes). El muestreo de las partículas se realizó de las 08:00 h a las 20:00 h y de las 20:00 h a las 08:00 h del día siguiente. Las concentraciones de las PST $\left(32.1,13.6,19.9,25.7\right.$ y $\left.38.9 \mu \mathrm{g} / \mathrm{m}^{3}\right)$ colectadas durante la noche en los cinco cruceros fueron aparentemente más altas que las colectadas durante el día $\left(18.4,15.6,16.4,23.6\right.$ y $\left.34.6 \mu \mathrm{g} / \mathrm{m}^{3}\right)$; sin embargo, al aplicar pruebas de hipótesis para evaluar estadísticamente si existían diferencias (a un nivel de confianza del 95\%), éstas no fueron significativas.

Por otra parte, al llevar a cabo la determinación de las PST, integrando diariamente las dos muestras de doce horas, su Norma Mexicana de Calidad del Aire, la cual fue de $260 \mu \mathrm{g} / \mathrm{m}^{3}$ en 24 horas (hasta junio de 2005), y actualmente de $210 \mu \mathrm{g} / \mathrm{m}^{3}$ en 24 horas, nunca se excedió.
\end{abstract}

Descriptores: Golfo de México, calidad del aire, contaminación del aire, partículas suspendidas totales.

\footnotetext{
Abstract

The University of Mex ico con ducted five re search cruises to in ves tigate the air qual ity over the Con ti nen tal Plat form of the Gulf of Mex ico. The first was con ducted in the sum mer of 1986 with the par tic ipa tion of the Na tional Oceanic and At mo spheric Ad min is tration
} 
(NOAA) of the United States, the Cen ter for At mo spheric Sci ences of the Na tional Univer sity of Mex ico and the Mex ican Navy, which fo cused on air qual ity mea surements. The other four were con ducted from Sep tem ber to Oc to ber and from No vember to De cem ber 2002, from Sep tem ber to Oc to ber 2003 and from May to June 2004, re spec tively, fo cused on air qual ity and ocean o graphic mea sure ments.

To tal sus pended par ticles (TSP) were deter mined by fol lowing the Mexican Official Stan dards and the United States Environ men tal Protection Agency Refer ence Method for To tal Sus pended Par ticles (High Vol ume Method). In or der to eval u ate the daily vari a tion in par tic u late, sam ples were col lected from 0800-2000 hand from 2000-0800 h lo cal time.

Night time TSP con cen tra tions for the five cruises were ap par ently higher than daytime val ues: $32.1,13.6,19.9,25.7$ and 38.9 mi cro grams per cu bic me ter, com pared to day time val ues of $18.4,15.6,16.4,23.6$ and 34.6 mi cro grams per cu bic me ter. However, not sig nif i cant differ ence was ob served be tween day time and night time. An a lyzing 12 hours TSP sam ples, two for each day, the Mex i can Air Qual ity Stan dard for TSP of 260 mi cro grams per cu bic me ter, during 24h (un til June 2005), and ac tually of $210 \mathrm{~g} / \mathrm{m}^{3}$ dur ing $24 \mathrm{~h}$, was never ex ceeded.

Key words: Gulfof Mexico, air qual ity, at mo spheric pollu tion, to tal sus pended particles.

\section{Introducción}

En los últimos años se ha enfatizado la importancia que representa el conocimiento de la química de la atmósfera a escala global. Temas tales como la disminución de la capa de ozono en la estratósfera, el transporte de contaminantes atmosféricos a través de grandes distancias y la influencia de sustancias, a nivel de trazas, sobre el clima, son hoy día tópicos de interés internacional. La solución a estos problemas requiere que se conozca, entre otras cosas, la química atmosférica a través de la determinación de contaminantes en los sitios de interés. Sin embargo, no tan sólo es importante desplazarse a estos lugares para realizar las determinaciones que se requieren, sino que éstas deben ser hechas mediante técnicas analíticas especificas, confiables y reproducibles.

En los meses de julio y agosto de 1986 se llevó a cabo un proyecto bilateral entre México y los Estados Unidos, con el objeto de estudiar la química de la atmósfera en el Golfo de México. Este crucero se inició en
Galveston, Texas, el 20 de julio de 1986, y se concluyó en Veracruz, México, el 23 de agosto de ese mismo año (Figura 1). La Secretaría de Marina de México proporcionó el buque oceanográfico $\mathrm{H}-02$ para llevar a cabo esta investigación. Los resultados obtenidos en este proyecto se encuentran documentados en el informe correspondiente, en el cual se llevaron a cabo determinaciones químicas, físicas, meteorológicas y oceanográficas para evaluar su variación temporal en el Golfo de México (Parungo y Miller, 1988 y Parungo et al., 1990).

Después de este proyecto de investigación, se realizaron cuatro proyectos más en la zona de la Plataforma Continental del Golfo de México: uno de septiembre a octubre de 2002 (Bravo et al., 2003a), otro de noviembre a diciembre del mismo año (Bravo et al., 2003b), el cuarto de septiembre a octubre de 2003 (Bravo et al, 2004a), y el último de mayo a junio de 2004 (Bravo et al., 2004b). Estos cuatro últimos cruceros fueron encabezados por el Instituto de Ciencias del Mar y Limnología de la Universidad Nacional Autónoma 
de México, con la participación y responsabilidad en la evaluación de la calidad del aire, de la Sección de Contaminación Ambiental, del Centro de Ciencias de la Atmósfera de la Universidad Nacional Autónoma de México. La UNAM proporcionó el buque de investigación oceanográfico "Justo Sierra", para el desarrollo de estos proyectos.
El itinerario seguido por el crucero de septiembre a octubre de 2002 se enfocó a la Plataforma Continental del Oriente del Golfo de México (Figura 2), mientras que el de noviembre a diciembre del mismo año, el de septiembre a octubre de 2003, y el de mayo a junio de 2004 se llevaron a cabo en la Plataforma Continental del Sur del Golfo de México (Figura 3).

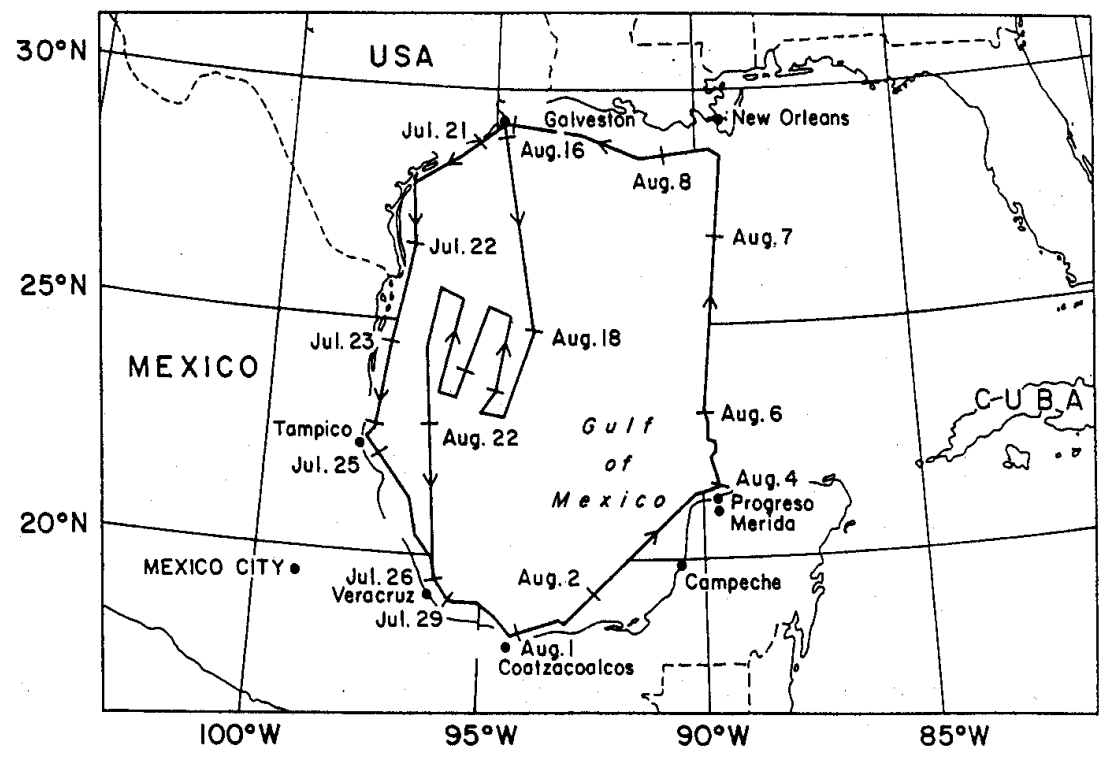

Figura 1. Derrotero del Crucero de Investigación del año 1986

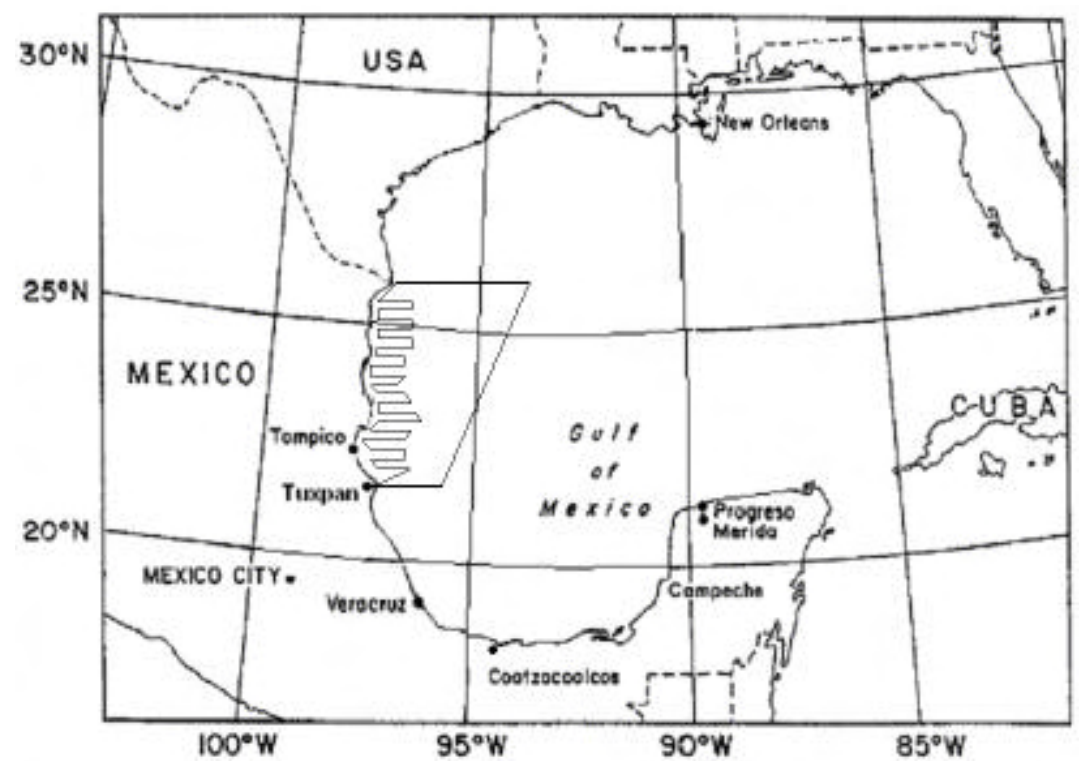

Figura 2. Derrotero del Crucero de Investigación de septiembre-octubre de 2002 


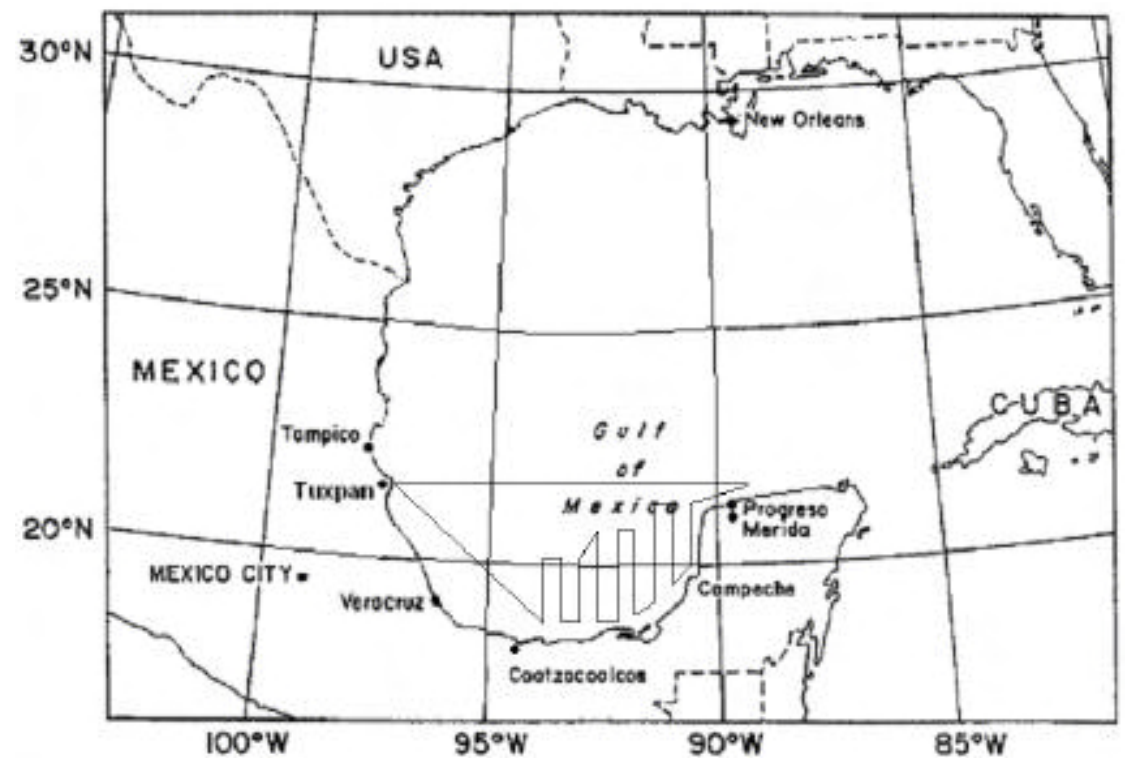

Figura 3. Derroteros de los Cruceros de investigación de noviembre-diciembre de 2002, septiembre-octubre de 2003 y de mayo-junio de 2004

\section{Metodología de muestreo y análisis}

La determinación gravimétrica de las partículas atmosféricas se llevó a cabo mediante métodos que cumplen con la Norma Oficial Mexicana (NOM-035, 1993), que lleva por título: "Método de Medición para Determinar la Concentración de Partículas Suspendidas Totales en el Aire Ambiente, y el Procedimiento para la Calibración de los Equipos de Medición", así como con el Método de Referencia de la Agencia de Protección Ambiental de los Estados Unidos (Manual Reference Method: 40 CFR Part 50, Appendix B) (Federal Register, 2000).

La colección y evaluación de las PST por el método de grandes volúmenes consiste en acondicionar y pesar el filtro antes (m1) y después del muestreo $\left(\mathrm{m}_{2}\right)$, a una temperatura y humedad predeterminadas. La diferencia en masa ( $\left.\mathrm{m}_{2}-\mathrm{m} 1\right)$ corresponde a la cantidad de partículas colectadas en el filtro. Debido a que la masa de las partículas suspendidas en el aire es pequeña, se debe colectar un gran volumen de aire. Por esta razón, se usó el muestreador de aire de grandes volúmenes (General Metal Works), el cual basa su funcionamiento en principios aerodinámicos para colectar partículas en el intervalo de 0.3 a $100 \mu \mathrm{m}$. Este intervalo de tamaños incluye por lo general, tanto a la fracción de las PST conocida como fracción fina (entre 0.1 y $2.0 \mathrm{~m}$ ) como a la fracción gruesa (entre 2.0 y poco más de $50 \mu \mathrm{m}$ ). En la figura 4 se presenta la distribución de las partículas en función de su diámetro aerodinámico (USEPA, 1996).

El muestreador consiste en un motor de succión y una pantalla de soporte para el filtro, así como de un transductor de presión y de un programador de tiempo. Los filtros de fibra de vidrio operan durante 12 ó 24 h, con un gasto volumétrico de $100 \mathrm{~m}^{3} \mathrm{~h}$.

La calibración se llevó a cabo usando el método de calibración por orificio (GMW-25) y un manómetro de agua. La masa final se determinó en el laboratorio, posteriormente, llevándose a cabo la custodia adecuada de los filtros. 
R. Sosa-E., H. Bravo-A., P. Sánchez-A., R. Soto-A., A. Alarcón-J. y J.D.W-Kahl

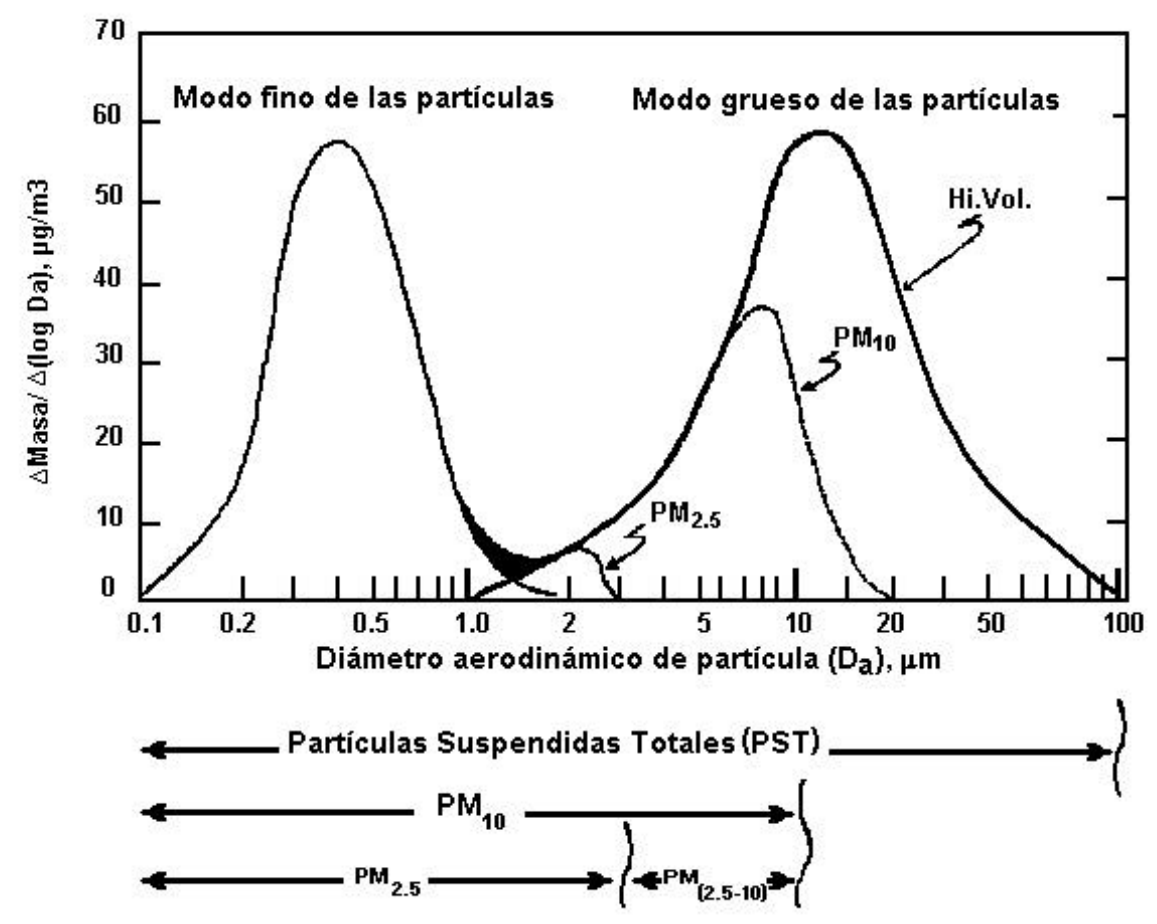

Figura 4. Esquema idealizado de los diferentes intervalos de tamaño de partículas colectadas por los sistemas de muestreo Hi. Vol. (PST), partículas iguales o menores a $10 \mathrm{~m}$ (PM10) y partículas menores o iguales a $2.5 \mathrm{~m}\left(\mathrm{PM}_{2.5}\right)$

Dependiendo del tipo de filtro utilizado, el intervalo de colección de partículas puede incrementarse hasta cerca de $100 \mu \mathrm{m}$. La masa de las partículas colectadas se determina por la diferencia de masas $\left(\mathrm{m}_{2}-\mathrm{m}_{1}\right)$, como se indicó antes, mientras que para determinar su concentración (expresada en $\left.\mu \mathrm{g} / \mathrm{m}^{3}\right)$, se debe conocer el volumen de aire que se filtró, en el intervalo de tiempo de muestreo, que por norma es de $24 \mathrm{~h}$. Para el caso específico de los cruceros de investigación, se llevó a cabo el muestreo cada 12 h, con el propósito de determinar la variación de las concentraciones de las partículas durante el día y la noche. Posteriormente, y para fines de comparación con la norma correspondiente para PST, se integraron las muestras del día y de la noche en una sola, para obtener un tiempo total de muestreo de 24 horas. La Figura 5 muestra el colector de las PST, ubicado a bordo del buque oceano- gráfico "Justo Sierra", en un sitio que reunió condiciones de seguridad, disponibilidad de energía eléctrica, y libre de obstáculos, con el propósito de obtener mediciones representativas. Por otra parte, el protocolo de la preparación y manejo de los filtros para el muestreo de las PST utilizado en la Sección de Contaminación Ambiental se presenta en la Figura 6. 
Determinación de partículas suspendidas totales durante cinco cruceros de investigación ...

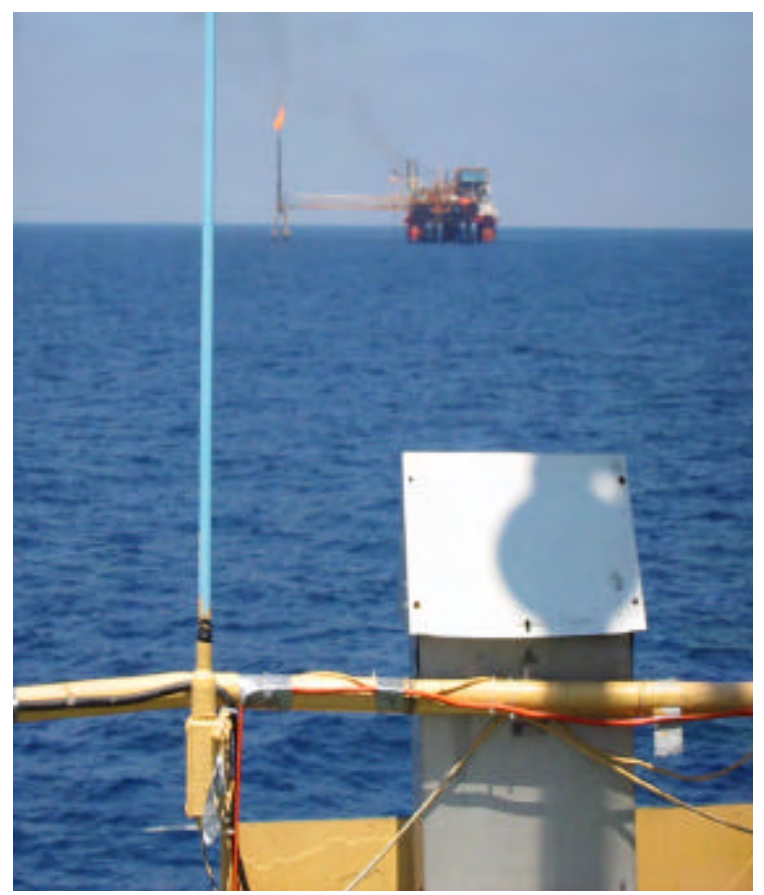

Figura 5. Muestreo de Partículas Suspendidas Totales en el Golfo de México

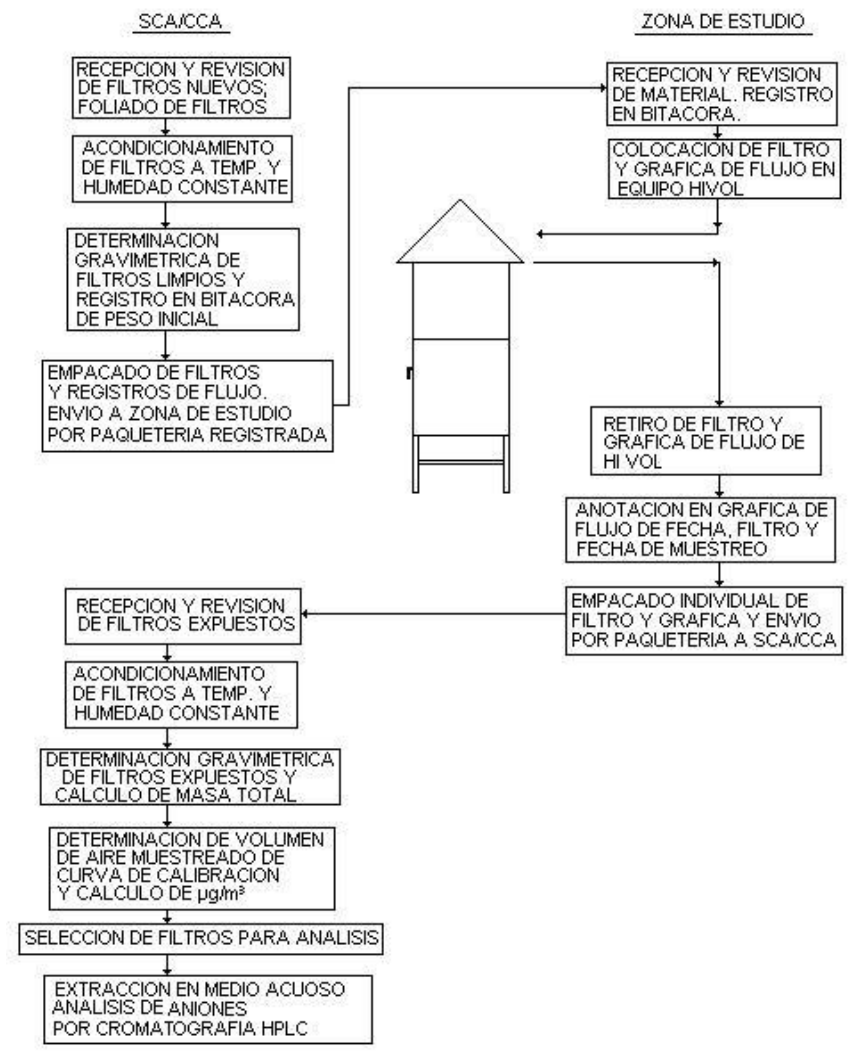

Figura 6. Protocolo del muestreo, manejo y análisis de filtros de partículas suspendidas totales colectadas por el método Hi. Vol 


\section{Resultados obtenidos}

Para evaluar la concentración de las partículas durante el día y durante la noche, las muestras se colectaron en dos períodos de muestreo: de las 08:00 a las 20:00 h y de las 20:00 a las 08:00 h tiempo local. Las concentraciones de las PST, para el día y para la noche, obtenidas durante los cinco cruceros de investigación, se presentan en la figura 7, mientras que las concentraciones promedio (día y noche) se indican en la tabla 1. En la figura 8, se representan, mediante diagramas de cajas, las concentraciones de las PST para los cinco cruceros (mínimo, máximo, percentiles 75,50 y 25$)$.

PST Muest reo Jul-Ago 1986

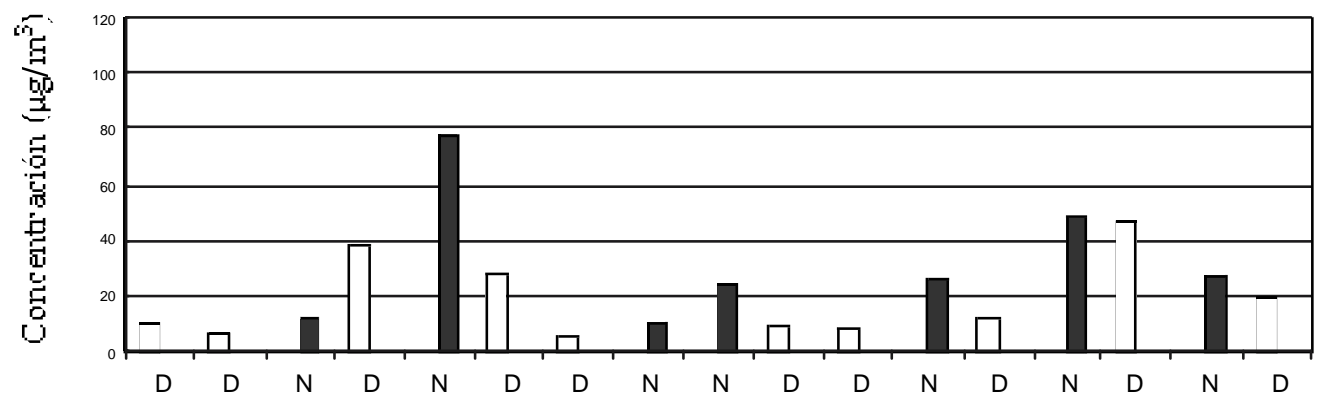

$7^{\mathrm{DIA}} \square^{\mathrm{NOCHE}}$

PST Muestreo- Se p-Oct 2002

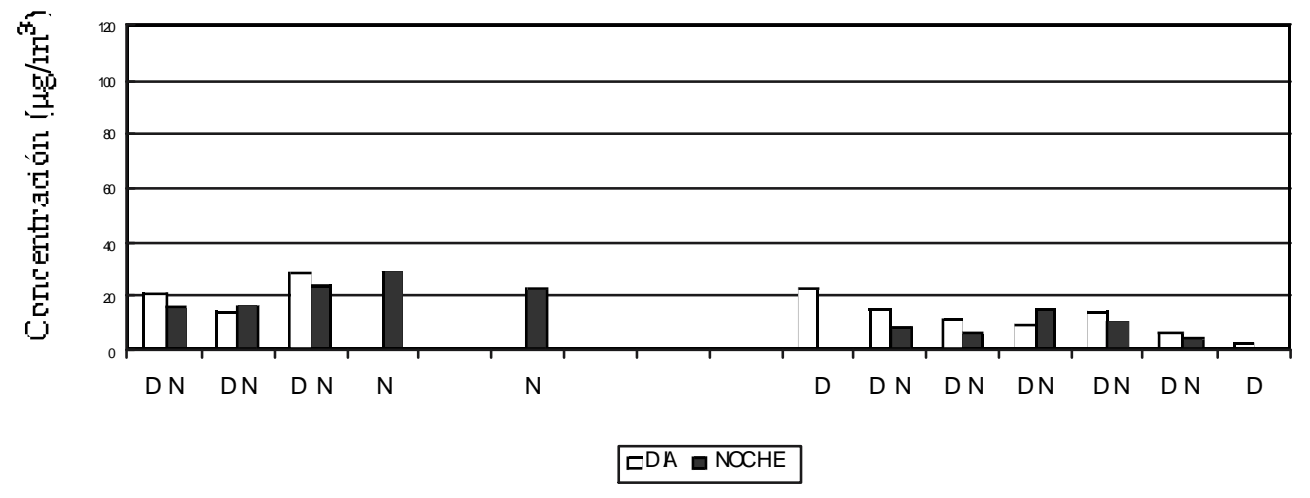

PST Mue streo - Nov-Dic 2002

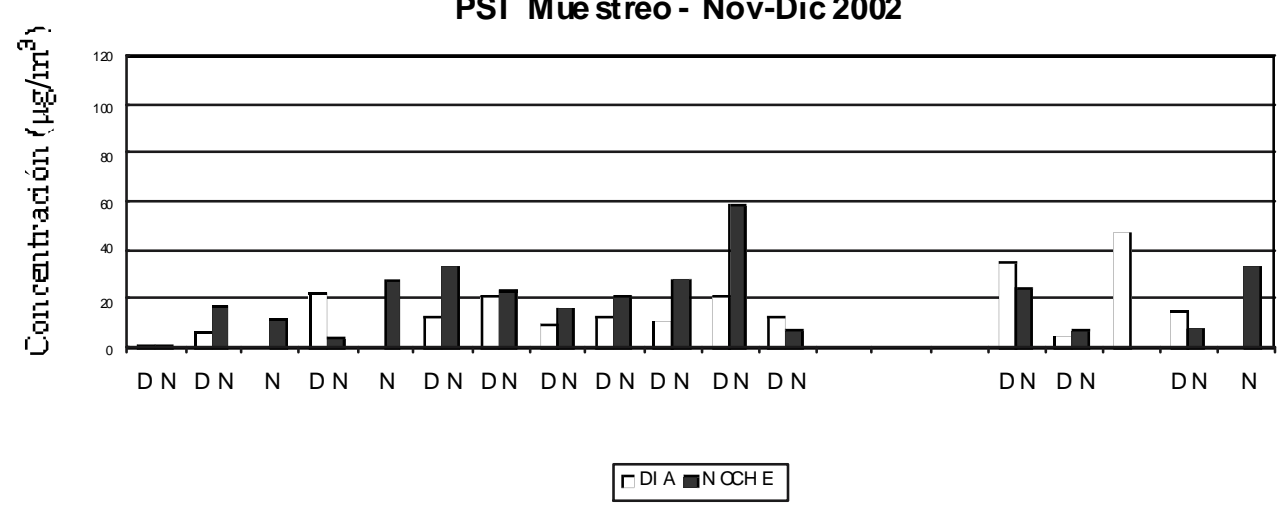

Figura 7. Concentraciones de Partículas Suspendidas Totales durante los cinco cruceros de investigación en el Golfo de México (continúa...) 
Determinación de partículas suspendidas totales durante cinco cruceros de investigación ...

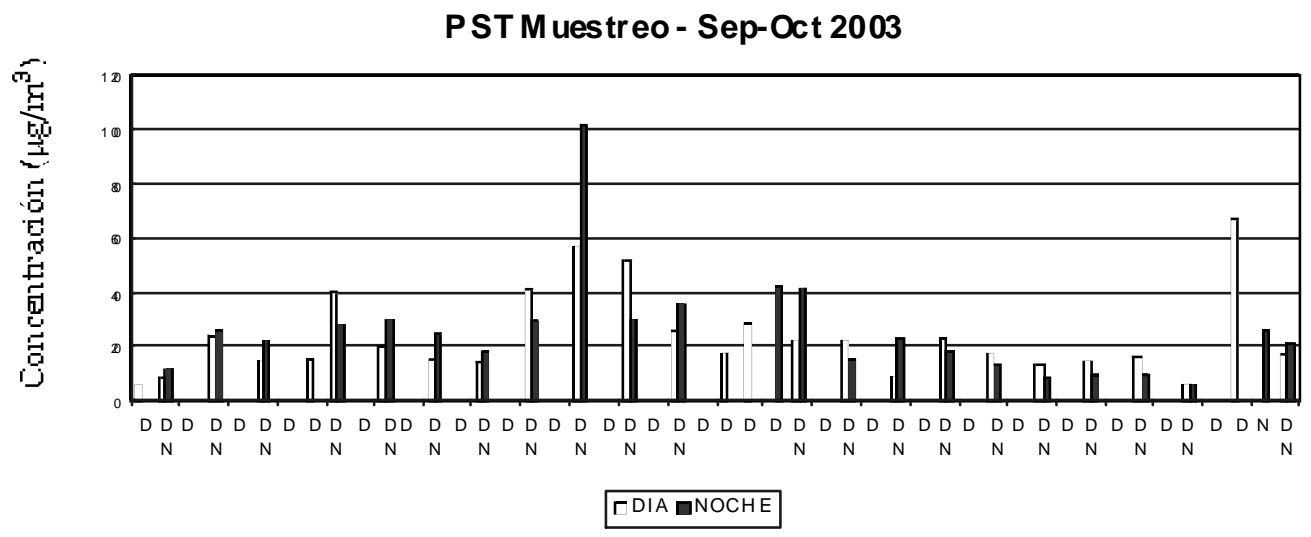

PST Muestr eo - May-Jun 2004

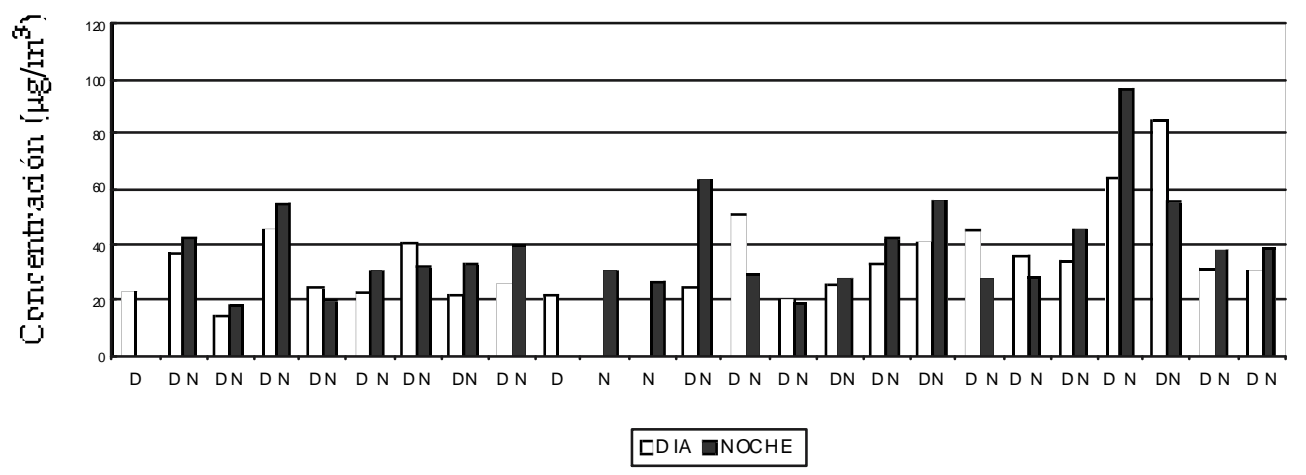

Figura 7. Concentraciones de Partículas Suspendidas Totales durante los cinco cruceros de investigación en el Golfo de México (... continuación)

Tabla 1. Concentraciones promedio de Partículas Suspendidas Totales en el Golfo de México

\begin{tabular}{ccc}
\hline Crucero & Concentración de PST & $\mu \mathrm{g} / \mathrm{m}^{3}$ \\
\hline & Día & Noche \\
\hline 1986 & $18.4 \pm 4.6$ & $32.1 \pm 9.0$ \\
Sept-Oct 2002 & $15.6 \pm 4.5$ & $13.6 \pm 4.8$ \\
Nov-Dic 2002 & $16.4 \pm 4.4$ & $19.9 \pm 5.0$ \\
Sept-Oct 2003 & $23.6 \pm 4.6$ & $25.7 \pm 5.5$ \\
May-Jun 2004 & $34.6 \pm 7.0$ & $38.9 \pm 8.1$ \\
\hline
\end{tabular}


R. Sosa-E., H. Bravo-A., P. Sánchez-A., R. Soto-A., A. Alarcón-J y J.D.W-Kahl

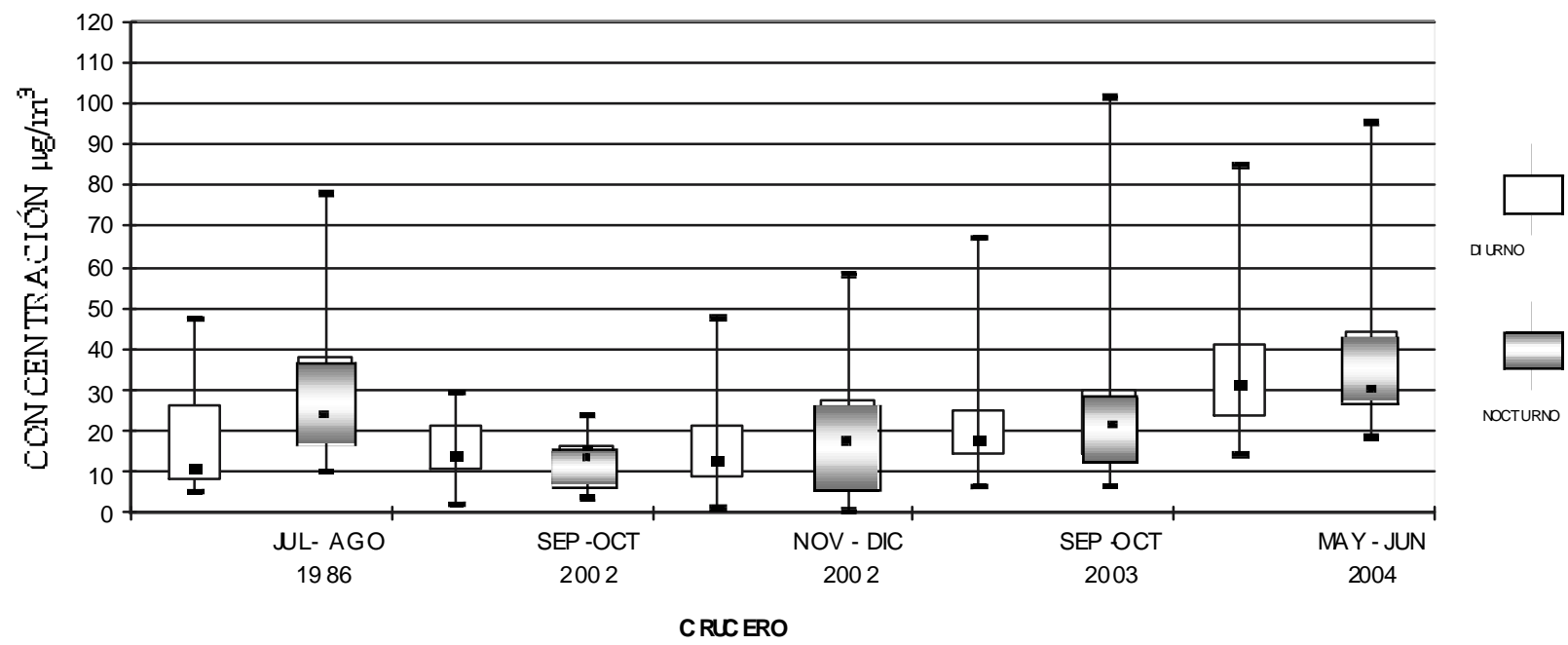

Figura 8. Representación mediante diagramas de caja de las concentraciones de Partículas Suspendidas Totales (PST) en el Golfo de México (máximo, mínimo percen tiles 25, 50 y 75 )

\section{Discusión}

Las concentraciones de las partículas muestreadas durante el día y durante la noche, en los cinco cruceros realizados, no fueron estadísticamente diferentes entre sí, al aplicar una prueba $t$ de hipótesis a un nivel de significancia del 5\%, aunque, puntualmente, en la mayoría de los casos, las concentraciones de dichas partículas hayan sido mayores en la noche en comparación con el día (32.1, 13.6, $19.9,25.7$ y $38.9 \mu \mathrm{g} / \mathrm{m}^{3}$ vs. $18.4,15.6,16.4,23.6$ y $\left.34.6 \mu \mathrm{g} / \mathrm{m}^{3}\right)$.

Para fines de comparación con la norma correspondiente para las PST, se integraron las muestras del día y de la noche en una sola, para obtener un tiempo de muestreo de 24 horas. El valor que establece la Norma Oficial Mexicana de Calidad del Aire para partículas suspendidas totales, que es de 260 $\mu \mathrm{g} / \mathrm{m} 3$ en 24 horas (hasta junio de 2005), y actualmente de $210 \mu \mathrm{g} / \mathrm{m} 3$ en 24 horas (NOM-025-SSA1, 1993), fue utilizado para fines de comparación con los resultados obtenidos, observándose que en ningún momento se rebasó.
El hecho de que las concentraciones puntuales de las partículas medidas en los cruceros hayan sido aparentemente mayores durante la noche, en comparación con el día, ha sido reportado en otros trabajos (Parungo y Miller, 1988; Bravo et al, 2003a y 2003b). En estas investigaciones se argumenta que la circulación de la brisa marina/terrestre, a lo largo de la costa del Golfo de México, es un factor que puede dar lugar a una distribución y a un transporte muy característicos de los contaminantes atmosféricos.

Debido a la gran capacidad térmica específica del agua, la temperatura de la superficie del mar cambia en menos de $2^{\circ} \mathrm{C}$ del día a la noche. La variación diurna de la temperatura del aire superficial sobre la tierra adyacente, por otra parte, puede ser tan grande como 10 a $20^{\circ} \mathrm{C}$. La gran diferencia de temperaturas a través de la línea costera crea una circulación térmica a nivel mesoescala conocida como circulación de la brisa marina / brisa terrestre.

Durante el día, la brisa marina se puede hacer presente a varios kilómetros en 
Determinación de partículas suspendidas totales durante cinco cruceros de investigación ...

latitudes tropicales, y transportar la masa de aire más fría a varios kilómetros tierra adentro. Las características físicas de la brisa marina se han investigado ampliamente en décadas recientes, de tal manera que ahora son bien conocidas (Arritt, 1992; Atkinson, 1981).

Las principales características de la brisa terrestre, son considerablemente menos conocidas. Pocos estudios se han enfocado a esto, debido a la dificultad que representa medir las características de los vientos en la costa.

Una revisión de la literatura sobre la brisa terrestre ha revelado lo siguiente:

- Típicamente penetra algunas decenas de kilómetros a través de la costa hacia el océano creando el potencial para el transporte de emisiones contaminantes generadas en tierra, hacia el océano por la noche (Atkinson, 1981; Lyons, 1974; Parameswaran et al, 1999, Stull, 1988).

- Se puede originar hasta $20 \mathrm{~km}$ tierra adentro (Banta et al, 1993).

- Puede penetrar hacia el mar de 50 a 80 km (Liu et al, 2002; Romero y Ramis, 1996; Zhong y Takle, 1993).

- Es más intensa a $20^{\circ}$ latitud norte (Xian y Pielke, 1991).

- Puede ser un mecanismo efectivo para transportar partículas atmosféricas generadas en la costa y depositarlas en la superficie del océano (Lu et al, 2003).

Otro factor que puede tener influencia sobre las diferentes concentraciones de las PST encontradas en los diferentes cruceros, es la presencia de lluvias. En el primer crucero se presentaron ocho eventos de lluvia, en el segundo, tercero y cuarto cruceros, se tuvieron tres, dos y doce eventos de lluvia, respectivamente (Bravo et al., 2006), mientras que en el último crucero, no se presentó ningún evento de lluvia, y fue en éste, en el que se observaron las mayores concentraciones de partículas (PST).

\section{Conclusiones y recomendaciones}

El valor que establece la Norma Oficial Mexicana de Calidad del Aire para partículas suspendidas totales, que es de 260 $\mu \mathrm{g} / \mathrm{m}^{3}$ en 24 horas (hasta junio de 2005), y actualmente de $210 \mu \mathrm{g} / \mathrm{m}^{3}$ en 24 horas, nunca fue excedida.

Las concentraciones promedio de las partículas suspendidas totales, medidas en los cinco cruceros en el Golfo de México, fueron, en su mayoría, ligeramente mayores durante la noche que durante el día.

La circulación de la brisa marina /terrestre es un factor que probablemente tenga influencia en los diferentes valores de concentraciones encontrados durante el día y durante la noche y que, en consecuencia, requiere ser estudiada con mayor detenimiento.

El último crucero correspondiente a mayo-junio de 2004 presentó los mayores niveles de PST, coincidiendo con un período de secas (no se tuvo ningún evento de lluvia).

Es necesario aumentar el número de días de muestreo durante el año, con el objeto de observar variaciones estacionales, ya que las determinaciones llevadas a cabo en este estudio se realizaron preponderantemente a mediados o finales de año, y se carece de información correspondiente a los primeros meses de cada año.

Con el objeto de lograr un mejor entendimiento acerca del transporte y dispersión de los contaminantes atmosféricos en la región del Golfo de México, la información obtenida en los cruceros de investigación se debe complementar con la información obtenida en diversas estaciones de monitoreo 
R. Sosa-E., H. Bravo-A., P. Sánchez-A., R. Soto-A., A. Alarcón-J y J.D.W-Kahl

localizadas en sitios estratégicos de la costa del Golfo de México.

\section{Agradecimientos}

Los autores agradecen a las siguientes personas su esfuerzo para llevar a cabo este trabajo: Pedro Hernández Nieto, Ruth Hernández Galicia y Jorge Santacruz Morhy, estudiantes de la Sección de Contaminación Ambiental del Centro de Ciencias de la Atmósfera; Armando Retama y M. en I. Mónica Jaimes Palomera, de la Red Automática de Monitoreo Atmosférico del Gobierno del Distrito Federal, y al Dr. Felipe Vázquez Gutiérrez (Responsable del Proyecto UNAM-PEMEX) del Instituto de Ciencias del Mar y Limnología de la UNAM.

\section{Referencias}

Arritt R.W. (1992). Effects of the LargeScale Flow on Characteristic Features of the Sea Breeze. J. Appl. Meteorol., Vol. 32, pp. 116-125.

Atkinson B.W. (1981). Meso-Scale Atmospheric Circulations. Academic Press, 495 pp.

Banta R.M., Olivier L.D. y Levinson D.H. (1993). Evolu tion of the Monterrey Bay Sea-Breeze Layer as Observed by Pulsed Doppler Lidar. J. Atmos. Sci., Vol. 50, pp. 3959-3982.

Bravo A.H., Sosa E.R., Sánchez A.P., Alarcón J.A.L., Jaimes P.M., Soto A.R. (2003a)."Evaluaciónde la contaminación atmosférica en el Oriente del Golfo de México", Campaña Oceanográfica OGM-6 (del 18 de septiembre al 3 de octubre de 2002). Sección de Contaminación Ambiental, Centro de Ciencias de la Atmósfera, Instituto de Ciencias del Mar y Limnología, UNAM. Bravo A.H., Sosa E.R., Sánchez A.P., Alarcón J.A.L., Jaimes P.M., Kahl D.W., Barrios R.J, Soto A.R., Bueno L.E., Gamboa A.R., Gamboa R.T., Abreu S.J. (2003b). “Evaluación de la contaminación atmosférica en el Sur del Golfo de México", Campaña Oceanográfica SGM-7 (NoviembreDiciembre de 2002). Sección de Contaminación Ambiental, Centro de Ciencias de la Atmósfera, Instituto de Ciencias del Mar y Limnología, UNAM. Bravo A.H., Sosa E.R., Sánchez A.P., Alarcón J.A.L., Jaimes P.M., Hernández N.P., Retama H.A., Sánchez R.M., Gómez R.C. Kahl D.W., Bueno L.E., Soto A.R. (2004a). "Eva- luación de la contaminación atmos- férica en el Sur del Golfo de México", Campaña Oceanográfica SGM-8 (Septiembre-Octubre de 2003). Sección de Contaminación Ambiental, Centro de Ciencias de la Atmósfera, Instituto de Ciencias del Mar y Limnología, UNAM. Junio.

Bravo A.H., Sosa E.R., Sánchez A.P., Alarcón J.A.L., Jaimes P.M., Santacruz M.J.D., Hernández N.P., Retama H.A., Sánchez R.M., Gómez R.C. Léon.D.I., Bueno L.E., Soto A.R. (2004b). "Evaluación de la Contaminación Atmosférica en el Sur del Golfo de México", Campaña Oceanográfica SGM-9 (mayojunio de 2004). Sección de Contaminación Ambiental, Centro de Ciencias de la Atmósfera, Instituto de Ciencias del Mar y Limnología, UNAM. Diciembre.

Bravo A.H., Sosa E.R., Kahl J., Sánchez A.P., Alarcón J.A.L., Soto A.R. (2006). "Acid Deposition Events during the Autumn of 2003 in a Research Cruise over the Gulf of Mexico". 99th Annual Conference \& Exhibition of the Air \& Waste Manage ment Asso ci a tion. New Orleans, Lousiana. June. Federal Register (2000). Reference Method for the Determination of Suspended Particulate Matter in the Atmosphere (High Volume Method). Fed. Reg. Appendix B, Part 50.

Liu K.Y., Wang Z. y Hsiao L.F. (2002). A Modeling of the Sea Breeze and its Impacts on Ozone Distribution in Northern Taiwan. Environmental Modelling and Soft ware, Vol. 17, pp. 21-27. 
Determinación de partículas suspendidas totales durante cinco cruceros de investigación ...

Lu R., Turco R.P., Stolzenbach K., Friedlander S.K., Xiong C., Schiff K., Tiefenthaler L. y Wang G.Y. (2003). Dry Deposition of Airborne Trace Metals on the Los Angeles Basin and Adja cent Coastal Waters, J. Geophysical Research-Atmospheres, Vol. 108, (D2).

Lyons W.A.(1974). Turbulent Diffusion and Pollutant Transport in Shoreline Environments. In: Lectures on Air Pollution and Environmental Impact Analysis. D.A. Haugen (Ed). Amer. Meteorol. Soc., pp. 136-208.

NOM-035-ECOL (1993). Norma oficial mexicana. Método de medición para determinar la concentración de partículas suspendidas totales en el aire ambiente y el procedimiento para la calibración de los equipos de medición.

NOM-025-SSA1 (1993). Norma oficial mexicana. Salud ambiental. Criterios para evaluar la calidad del aire ambiente, con respecto a mate rial particulado. Valor de concentración máxima de material particulado para partículas suspendidas totales, partículas menores de 10 micrómetros y partículas menores de 2.5 micrómetros en el aire ambiente como medida de protección a la salud de la población. Con modificaciones al 13 de junio de 2005.

Parameswaran K., Nair P.R., Rajan R. y Ramana M.V. (1999). Aerosol Loading in Coastal and Marine Environments in the Indian Ocean Region During Winter Season. Current Science, Vol. 76, pp. 947-955.

Parungo F. y Miller J.M. (1988). Air Chem istry Studies Over the Gulf of Mexico. NOAA Tech. Memo. ERL/ESG-29, Boulder, CO.

Parungo F., Nagamoto C., Hoyt S. y Bravo A.H. (1990). The Investigation of Air Quality and Acid Rain Over the Gulf of Mexico. Atmospheric Environment. Vol. 24A(1), pp. 109-123.

Romero R. y Ramis C. (1996). A Numerical Study of the Transport and Diffu sion of Coastal Pollutants During the Breeze Cycle in the Island of Mallorca. Annales Geophysicae, Vol. 14, pp. 351-363.

Stull R.B. (1988). An Introduction to Boundary Layer Meteorology. Kluwer Academic Publishers, Boston, 666 pp. U.S.EPA (1996). Air Quality Criteria for Particulate Matter. Vol. II, pp. 10-185.

Xian Z. y Pielke R.A. (1991). The Effects of Width of Land masses on the Devel opment of Sea Breezes. J. Appl. Meteorol., Vol. 30, pp. 1280-1304.

Zhong S. y Takle E.S. (1993). The Effects of Large-Scale Winds on the Sea-LandBreeze Circulations in an Area of Complex Coastal Heating. J. Appl. Meteorol., Vol. 32, pp. 1181-1195. 
R. Sosa-E., H. Bravo-A., P. Sánchez-A., R. Soto-A., A. Alarcón-J y J.D.W-Kahl

\section{Semblanza de los autores}

Rodolfo Sosa-Echeverría. Realizó su licenciatura en ingeniería química, la maestría en ingeniería ambiental y el doctorado en ingeniería en la UNAM. Labora en la Sección de Contaminación Ambiental del CCA desde 1986, siendo actualmente Investigador. Desde 1988 a la fecha ha impartido la cátedra de muestreo y análisis del aire en el posgrado en ingeniería ambiental de la Facultad de Ingeniería. En 1994, asesoró al Ministerio de Desarrollo Sostenible y Medio Ambiente de Bolivia en la realización del Reglamento en Materia de Contaminación atmosférica como parte de la Ley de Medio Ambiente. Es perito colegiado del Colegio N acional de Ingenieros Químicos y de Químicos (CONIQQ) en protección ambiental y fue presidente del Capítulo México de la Air and Waste Manage ment Asso ci a tion, de 1991 a 1993. Es miembro del Sistema Nacional de Investigadores.

Humberto Bravo-Alvarez. Realizó su licenciatura en química en la UNAM, así como la maestría en química nuclear.

Posteriormente, obtuvo el grado de doctor en ingeniería en la Universidad de West Virginia. Es investigador en el Centro de Ciencias de la Atmósfera (CCA) de la UNAM, donde se desempeña como jefe de la Sección de Contaminación Ambiental desde 1977. Su área de interés por más de 30 años ha sido la contaminación y la ingeniería ambiental. Ha sido profesor de asignatura en la maestría en ingeniería ambiental en la División de Estudios de Posgrado de la Facultad de Ingeniería (DEPFI) de la UNAM desde 1988. Es perito colegiado del Colegio Nacional de Ingenieros Químicos y de Químicos (CONIQQ) en protección ambiental, así como Qualified Envi ron mental Profes sional (QEP) del Insti tute of Profes sional Envi ron mental Prac tice.

Pablo Sánchez-Alvarez. Realizó la licenciatura en biología en la Facultad de Ciencias de la UNAM. Actualmente labora en la Sección de Contaminación Ambiental del CCA. Es responsable de la operación y mantenimiento de la estación de monitoreo automático de contaminantes criterio y deposición ácida. Es instructor certificado en la línea de investigación de la atmósfera y el clima del Programa Internacional de Aprendizajes y Observaciones Globales en Beneficio del Medio Ambiente (The GLOBE Program México).

Rogelio Soto-Ayala. Realizó su licenciatura en química y su maestría en química analítica en la Facultad de Química de la UNAM. Obtuvo el doctorado en ingeniería ambiental en la División de Estudios de Posgrado de la Facultad de Ingeniería de la UNAM. Realizó un posdoctorado en la Universidad de Córdoba en España, y una estancia de investigación por tres meses, en el Instituto de Investigaciones en la Tropósfera en Leipzig, Alemania. Tiene 26 años de experiencia docente en la División de Ciencias Básicas de la Facultad de Ingeniería, impartiendo las asignaturas de Química, Termodinámica, Fisicoquímica y Física Exper i mental. Ha impartido múltiples cursos para profesores, fundamentalmente en el área de la Química y la Termodinámica. Colabora en la Sección de Contaminación Ambiental del Centro de Ciencias de la Atmósfera en actividades de investigación.

Ana Luisa Alarcón-Jiménez. Realizó la licenciatura de química en alimentos en la Facultad de Química de la UNAM. Actualmente tiene a su cargo el laboratorio de análisis químico en la Sección de Contaminación Ambiental del CCA, donde participa en el desarrollo de diversos proyectos de investigación, mediante la utilización, entre otras técnicas, de la cromatografía de líquidos de alta resolución.

Jonathan D.W.-Kahl. Realizó su doctorado en Ciencias Atmosféricas en la Universidad de Michigan. Su posdoctorado en el National Research Council de la National Oceanic and Atmospheric Administration (NOAA). Desde 1990 es profesor de ciencias atmosféricas en el Departamento de Ciencias Matemáticas en la Universidad de Wisconsin-Milwaukee, Milwaukee, Wisconsin. Ha realizado estancias cortas y ha sido profesor invitado para impartir conferencias en el Centro de Ciencias de la Atmósfera de la UNAM. 